\title{
Which biologic agents increase perioperative complications in patients with inflammatory bowel disease?
}

\author{
Jihye Park ${ }^{1,2}$ \\ ${ }^{1}$ Department of Internal Medicine and ${ }^{2}$ Institute of Gastroenterology, Yonsei University College of Medicine, Seoul, Korea
}

\begin{abstract}
Article: Vedolizumab does not increase perioperative surgical complications in patients with inflammatory bowel disease, cohort study (Intest Res 2021;20:72-77)
\end{abstract}

The medical treatment of patients with inflammatory bowel disease (IBD) has changed over the last decades with a significant increase in use of biologic agents, including anti-tumor necrosis factor (TNF)- $\alpha$ inhibitors, anti- $\alpha 4 \beta 7$-integrin inhibitors, and anti-interleukin 12/23 inhibitors. ${ }^{1}$ Nevertheless, certain patient group with aggressive disease course required IBD-related intestinal surgery in real world setting., ${ }^{2,3}$ The perioperative complications could be increased in patients with previous corticosteroid use, malnutrition, intra-abdominal abscesses, and previous surgical history. Also, the impact of biologic agents treatment on perioperative complications were investigated in many previous studies. ${ }^{5}$

The anti-TNF- $\alpha$ inhibitors, which are the first biologic agents, could not only achieve induction and maintenance of remission of IBD, but also regulate the intestinal wound healing process. Because of the potent concerns about delaying wound healing during the perioperative period, several retrospective studies, meta-analyses, and prospective studies assessed the perioperative complications in IBD patients treated with antiTNF- $\alpha$ inhibitors. ${ }^{5}$ Brouquet et al. ${ }^{6}$ reported that patients treated with anti-TNF within 12 weeks of an IBD-related intestinal surgery were positively associated with postoperative morbid-

Received December 14, 2021. Accepted December 20, 2021. Correspondence to Jihye Park, Department of Internal Medicine, Yonsei University College of Medicine, 50-1 Yonsei-ro, Seodaemungu, Seoul 03722, Korea. Tel: +82-2-2228-1976, Fax:+82-2-393-6884, E-mail: wisdompark@ yuhs.ac ity in the prospective nationwide cohort study (odds ratio, 1.99; $95 \%$ confidence interval, $1.17-3.39 ; P=0.011$ ). In contrast to this study, Cohen et al. ${ }^{7}$ reported that the perioperative complications were not different between patients treated with anti-TNF- $\alpha$ inhibitors and controls (20\% vs. $19.4 \%)$. Overall, the relationship between anti-TNF- $\alpha$ inhibitors and perioperative complications in patients with IBD remains controversial.

Vedolizumab, an approved biologic agent after anti-TNF- $\alpha$ inhibitors, blocks leucocyte trafficking limited to the inflamed intestine. Although gut-specific mechanisms of action reduce the risk of serious or opportunistic infections, the concerns have been raised that the lack of leukocytes may delay the perioperative intestinal wound healing process. Furthermore, the rates of perioperative complications in patients treated with vedolizumab were investigated compared to the patient treated with anti-TNF- $\alpha$ inhibitors.

Lightner et al. ${ }^{8}$ reported that the 30-day postoperative surgical site infection (SSI) rates were significantly higher in patient treated with vedolizumab $(\mathrm{n}=94)$ within 12 weeks of an IBDrelated intestinal surgery as compared to patients treated with anti-TNF- $\alpha$ agents $(\mathrm{n}=129)$ or no biological therapy $(\mathrm{n}=172)$ ( $37 \%$ vs. $10 \%$ vs. $13 \%$ ), and vedolizumab use was an independent risk factor of SSI in the multivariable analysis. Superficial SSI ( $6 \%$ vs. $4 \%$ vs. $21 \%$ ), deep space SSI ( $6 \%$ vs. $5 \%$ vs. $14 \%$ ), and mucocutaneous separation ( $1 \%$ vs. $1 \%$ vs. $7 \%$ ) complications were significantly increased in patient treated with vedolizumab compared to patients treated with anti-TNF- $\alpha$ or 
no biological therapy. ${ }^{8}$ In contrast to this study, Park et al. ${ }^{9}$ reported that the 30-day postoperative SSI rates were not different in patients treated with vedolizumab $(\mathrm{n}=94)$ within 30 days of an IBD-related intestinal surgery as compared to patients treated with anti-TNF- $\alpha(n=92)$ ( $14.9 \%$ vs. $12.0 \%)$. Systemic reviews and meta-analysis revealed no differences regarding to the perioperative complications exposed to vedolizumab versus anti-TNF- $\alpha$ agents. ${ }^{5}$

In the current issue, Poylin et al. ${ }^{10}$ reported the perioperative complication at both 30 days and 90 days in patients treated with vedolizumab $(\mathrm{n}=38)$ and anti-TNF- $\alpha$ agents $(\mathrm{n}=94)$ within 4 weeks of surgery to include higher biologic agent levels at the time of surgery. The SSI at 30 days (7.9\% vs. $7.4 \%)$ and 90 days ( $0 \%$ vs. $2.1 \%)$ complications were not different in patient treated with vedolizumab compared to patients treated with anti-TNF- $\alpha$ agents in this study. ${ }^{10}$ In the multivariate analysis, the immunomodulator use or biologic agents were not associated with risk of perioperative complications. ${ }^{10}$ The hypoalbuminemia $(<3.6 \mathrm{~g} / \mathrm{dL})$ was the only significant risk factor associated with the risk of overall perioperative complications. ${ }^{10}$

The short-term and long-term perioperative complication rates were low in patients treated with biologic agents within 4 weeks before surgery. A prospective study with strong evidence is needed to overcome the controversy over the risk of perioperative complication risks on biologic agents. In addition, the multidisciplinary team approach for the intestinal surgery of IBD patients should consider individual risk factors, including nutritional status.

\section{ADDITIONAL INFORMATION}

\section{Funding Source}

The author received no financial support for the research, authorship, and/or publication of this article.

\section{Conflict of Interest}

Park $\mathrm{J}$ is an editorial board member of the journal but was not involved in the peer reviewer selection, evaluation, or decision process of this article. No other potential conflicts of interest relevant to this article were reported.

\section{Author Contribution}

Writing and approval of final manuscript: Park J.

\section{ORCID}

Park J

https://orcid.org/0000-0002-5836-8735

\section{REFERENCES}

1. Barberio B, Savarino EV, Card T, et al. Incidence comparison of adverse events in patients with inflammatory bowel disease receiving different biologic agents: retrospective longterm evaluation. Intest Res 2022;21:114-123.

2. Murthy SK, Begum J, Benchimol EI, et al. Introduction of antiTNF therapy has not yielded expected declines in hospitalisation and intestinal resection rates in inflammatory bowel diseases: a population-based interrupted time series study. Gut 2020;69:274-282.

3. Song EM, Yang SK. Natural history of inflammatory bowel disease: a comparison between the East and the West. Intest Res 2021 Dec 2 [Epub]. https://doi.org/10.5217/ir.2021.00104.

4. Huang W, Tang Y, Nong L, Sun Y. Risk factors for postoperative intra-abdominal septic complications after surgery in Crohn's disease: a meta-analysis of observational studies. J Crohns Colitis 2015;9:293-301.

5. Quaresma AB, Yamamoto T, Kotze PG. Biologics and surgical outcomes in Crohn's disease: is there a direct relationship? Therap Adv Gastroenterol 2020;13:1756284820931738.

6. Brouquet A, Maggiori L, Zerbib P, et al. Anti-TNF therapy is associated with an increased risk of postoperative morbidity after surgery for ileocolonic Crohn disease: results of a prospective nationwide cohort. Ann Surg 2018;267:221-228.

7. Cohen BL, Fleshner P, Kane SV, et al. Anti-tumor necrosis factor therapy is not associated with post-operative infection: results from prospective cohort of ulcerative colitis and Crohn's disease patients undergoing surgery to identify risk factors for postoperative infection I (PUCCINI). Gastroenterology 2019;156(6 Suppl 1): S-80.

8. Lightner AL, McKenna NP, Tse CS, Raffals LE, Loftus EV Jr, Mathis KL. Postoperative outcomes in vedolizumab-treated Crohn's disease patients undergoing major abdominal operations. Aliment Pharmacol Ther 2018;47:573-580.

9. Park KT, Sceats L, Dehghan M, et al. Risk of post-operative surgical site infections after vedolizumab vs anti-tumour necrosis factor therapy: a propensity score matching analysis in inflammatory bowel disease. Aliment Pharmacol Ther 2018; 48:340-346.

10. Poylin VY, Serrato JC, Pastrana Del Valle J, Feuerstein JD. Vedolizumab does not increase perioperative surgical complications in patients with inflammatory bowel disease, cohort study. Intest Res 2022;21:72-77. 